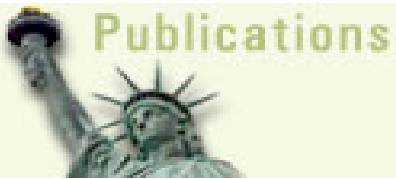

American Journal of Biochemistry and Biotechnology 3 (4): 207-215, 2007

ISSN 1553-3468

(C) 2007 Science Publications

\title{
Creation of Tetracycline Regulatable Knockouts of ORFF Gene from the LD1 Locus of Leishmania donovani
}

\author{
${ }^{1}$ Bindu Sukumaran, ${ }^{1}$ Manju Jain, ${ }^{2}$ Peter J. Myler, ${ }^{2}$ Kenneth. D. Stuart and ${ }^{1}$ Rentala Madhubala \\ ${ }^{1}$ School of Life Sciences, Jawaharlal Nehru University, New Delhi 110067, India \\ ${ }^{2}$ Seattle Biomedical Research Institute, 307 Westlake Ave N, Seattle, WA 98109-5219, USA
}

\begin{abstract}
The tetracycline repressor/operator system was adapted for use in Leishmania donovani. In order to investigate the possible importance and function of open reading frame $F$ (ORFF) in Leishmania donovani strain AG83 that has a single copy of ORFF in the LD1 locus on chromosome 35 , we have attempted to generate tetracycline regulatable single allele mutants of ORFF. Leishmania cells expressing the tetracycline repressor were transfected with tetracycline inducible vector expressing ORFF gene and having hygromycin as the selectable marker. The first endogenous genomic copy of ORFF was knocked out by homologous replacement with an inducible copy. Single ORFF gene disruption having an inducible copy of ORFF did not affect the growth of the parasites. This study, even though not conclusive, shows the possibility of generating functional tetracycline regulatable single knock out mutants of Leishmania donovani. The present study also demonstrates that ORFF gene might be an important gene and may be involved in various functions like attachment of Leishmania to the macrophages.
\end{abstract}

Key words: $\quad$ Infectivity; knockout; LD1 locus; Leishmania donovani; ORFF gene; tetracycline inducible

\section{INTRODUCTION}

Leishmania are protozoan parasites of the order Kinetoplastida that cause a variety of diseases in humans, from dermal lesions to visceral infections and are widespread in the tropics and subtropics. Current options for disease control and treatment are limiting The Leishmania genome project has identified new genes at a rapid rate. The 32.8-megabase haploid genome of Leishmania major (Friedlin strain) is published. By sequence homology, the products of many of these genes are predicted to participate in a variety of physiological activities and regulatory processes. However, the functions of a substantial fraction of these protein-coding genes are unknown. Unlike conventional gene replacement strategy, an inducible system that provides stringent regulation of gene expression in Leishmania offers great advantage for studying gene functions, particularly for essential genes and those encoding toxic products ${ }^{[1]}$.

The tetracycline repressor/operator system has been used to regulate transcription in yeast, plants and eukaryotes $^{[2-4]}$. There is as yet no reproducible evidence of a bonafide pol II promoter in Trypanosomatids ${ }^{[5,6]}$. Nevertheless studies have shown that the rRNA promoter is capable of directing efficient expression of a protein-coding gene by RNA polymerase I in these organisms $^{[7-11]}$. Tetracycline inducible expression system employing a polI-like promoter has been used successfully in Trypanosoma. brucei, which allowed the precise control of the expression of introduced genes through a range of four orders of magnitude. The rRNA promoter from Leishmania donovani is well characterized ${ }^{[10]}$. Yan et $a l^{[1]}$ have adapted an inducible promoter system that allows expression of proteincoding genes to be regulated by a tetracycline responsive rRNA promoter in $L$. donovani. In this system, the tetracycline-responsive repressor (TETR) binds to the tetracycline-responsive operator (TetO) close to the transcription initiation site (TIS) and prevents transcription ${ }^{[12]}$. Transcription is induced by addition of tetracycline, which binds to the repressor, causing it to dissociate from the operator, thereby allowing transcription to proceed.

A second generation tetracycline-responsive repressor-operator system has been generated earlier ${ }^{[6]}$. In the second-generation system, the selection marker $(H Y G)$ and the test gene $(L U C)$ are placed on opposite DNA strands and are driven by different promoters ${ }^{[6]}$. In this system, expression of reporter luciferase gene $(L U C)$ is driven by the inducible Leishmania ribosomal

Corresponding Author: Professor R. Madhubala, School of Life Science, Jawaharlal Nehru University, New Delhi-110067, India. Fax: 91-11-26106630 
RNA promoter on the DNA strand opposite to a hygromycin resistance gene $(H Y G)$ whose expression is driven by the endogenous pol I promoter (rDNA locus) or the endogenous pol II promoter (LD1 locus). We have attempted to generate tetracycline regulatable mutants of ORFF gene in Leishmania donovani using this second generation tetracycline-responsive repressor-operator system.

The multigene LD1 locus is present on chromosome 35 in all Leishmania isolates examined ${ }^{[13-}$

${ }^{18]}$. This sequence is amplified in approximately $15 \%$ of all Leishmania strains examined ${ }^{[18]}$. This amplification occurs in the absence of an intentional drug selection, suggesting that it has an important role. The gene ORFF from the LD1 locus encodes $\sim 32 \mathrm{kDa}$ protein which is localized in the nucleus ${ }^{[13]}$. This gene is universally amplified in all strains of Leishmania showing LD1 amplification, suggesting that it may provide a selective advantage for the parasite survival. Recombinant ORFF protein (rORFF) has been found to be a differential diagnostic and a promising vaccine candidate $^{[17,19-23]}$. The protein product of this gene does not have sequence homology to any known protein in the database indicating that it may be a Leishmania specific gene product.

In order to study the possible importance and function of ORFF gene, we have attempted to generate tetracycline regulatable mutants of ORFF in Leishmania donovani strain AG83 that has a single copy of ORFF in the LD1 locus on chromosome 35. Leishmania cells expressing the tetracycline repressor were transfected with tetracycline inducible vector expressing ORFF gene and having hygromycin as the selectable marker. These genetically engineered ORFF mutants of Leishmania were characterized for the scorable changes in their phenotype.

\section{MATERIALS AND METHODS}

Parasite cell culture: All parasite cell lines used in this study were derived from an Indian isolate of $L$. donovani AG83 (MHOM/IN/1983/AG83). Promastigotes were grown at $22^{\circ} \mathrm{C}$ in M199 medium (Sigma) with $10 \%$ fetal calf serum (FCS) (GIBCO, BRL).

Plasmid constructs: Plasmid constructs that were used for transfections are shown in Figure 1. The first construct pTupactet ${ }^{[1,6]}$ was used to insert the TETR gene into the $\alpha$-tubulin locus of $L$. donovani (Figure
1A). pTupactet contains the puromycin $\mathrm{N}$-acetyl transferase $(P A C)$ gene flanked by the $5^{\prime}$ and $3^{\prime}$ intergenic regions from the L. major
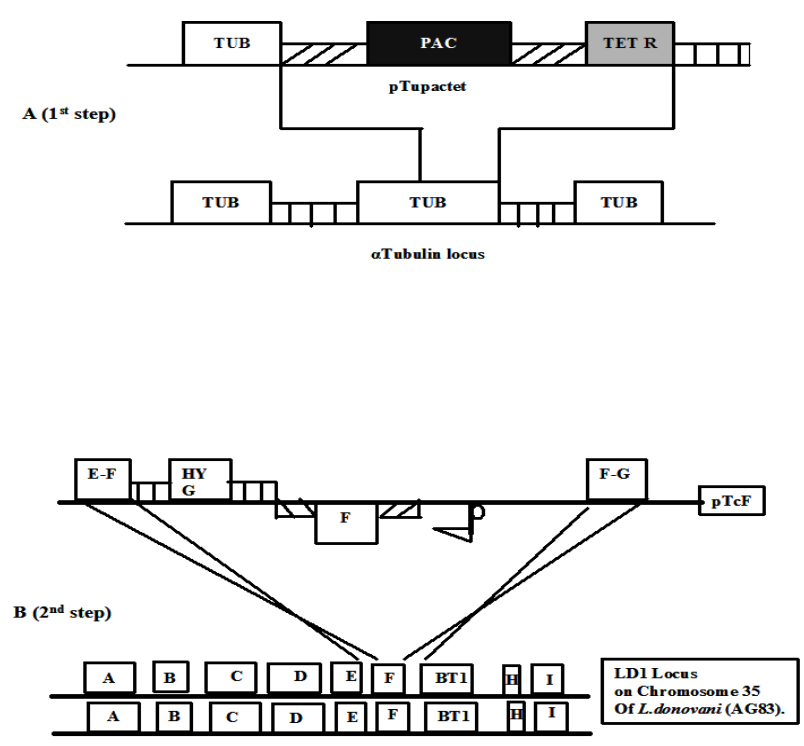

Fig. 1: Steps involved in the creation of tetracycline regulatable knockout of ORFF and the plasmid constructs used in targeted gene replacement experiments. A: Construct pTupactet used to introduce TetR into the tubulin locus. B: Construct $\mathrm{pTcF}$ used to replace first allele of ORFF with tetracycline regulatable allele of ORFF.

dihydrofolate reductase/thymidilate synthetase (DHFRTS) gene ${ }^{[24]}$ and the Tn10-encoded TETR gene ${ }^{[4]}$ (Figure 1A). The 5' and 3' DHFR-TS intergenic regions provide the splice leader sequence and the poly $\mathrm{A}^{+}$tail for the expression of selectable markers.

Generation of pTcF. Plasmid pFHLTG that had the $L U C$ gene under the control of a tetracycline-inducible $\mathrm{P}_{\text {RRNA }}$, downstream and on the opposite strand of $H Y G$ was used in the present study to generate the construct $\mathrm{pTcF}^{[1,6]}$ (Figure 1B). ORFG also known as BT1 has been mentioned in Figure 1B as BT1.

Three sub-cloning steps were involved in the construction of $\mathrm{pTcF}$ from $\mathrm{pFHLTG}$.

1. $F-G$ intergenic region was amplified from AG83 genomic DNA by PCR using primers 5'ApaFG ACGTGGGCCCTAAGCGCCTCGCCTATACC and 3'KHdFG - 


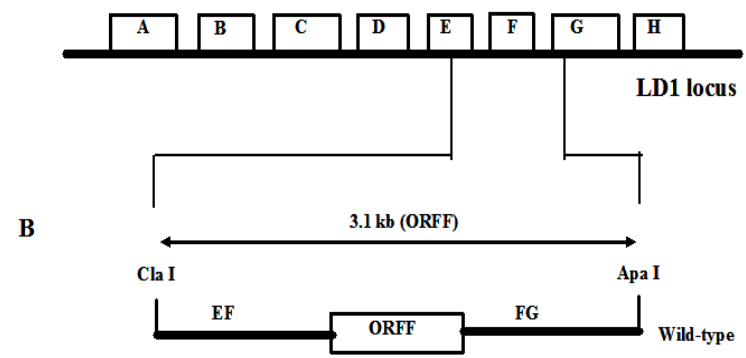

C

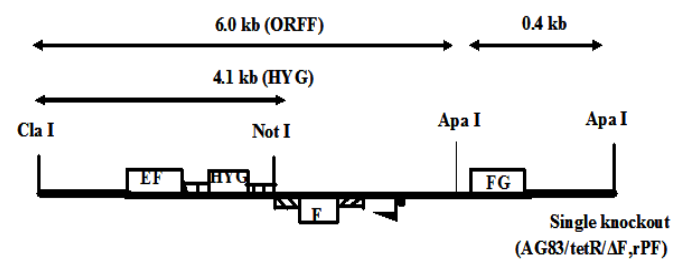

Fig. 2: Schematics of the ORFF genomic region before and after the targeted deletions of ORFF gene. The various probes giving the expected band size is given in brackets. A: schematic representation of LD1 genomic locus. B: ClaI and ApaI fragment in wild type AG83. C: Changes in ClaI and ApaI restriction-fragment size following $\mathrm{HYG}$ construct integration $(\mathrm{pTcF})$ are indicated. The size of the ClaI and NotI restriction- fragment obtained is also shown.

\section{ACGTGGTACCAAGCTTAGTCGCATCAGCCCAT}

TG. The 432-bp PCR product obtained was purified, digested with $A p a \mathrm{I}$ and $K p n \mathrm{I}$ and cloned into ApaI and $K p n I$ digested plasmid pFHLTG. The clone pFHLT-FG of size $10.76 \mathrm{~kb}$ was used for the next sub-cloning step. 2. E-F intergenic region was amplified from AG83 genomic DNA by PCR using primers 5'BstEFCTGCAGAACCAATGCATTGGCGTGCCTGTGTG CGTAAG and 3 'AscEF ACTGGCGCGCCTACAGCAGGAGCTACATAAAC G. The 340-bp PCR product obtained was purified, digested with BstXI and $A s c$ I and cloned into BstXI and $A s c$ I digested plasmid pFHLT-FG. The clone pEFHLT-FG $(10.2 \mathrm{~kb})$ was used for the third sub-cloning step.

3. ORFF was amplified from AG83 genomic DNA by PCR using primers 5 'BamF -

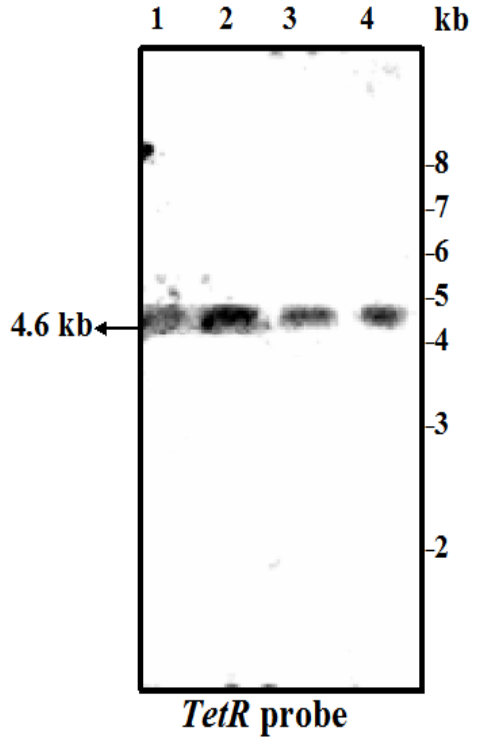

Fig. 3: Genomic Southern blot analysis of AG83/tetR clones using TetR probe (647 bp). Genomic DNA was isolated from AG83/tetR clones and was digested with HindIII. The DNA was size fractionated on $1 \%$ agarose gel and blotted to nylon membrane. The filters were hybridized with ${ }^{32}$ P-labeled TetR probe. Filters were washed and exposed overnight to X-ray film. Lanes 1, 2, 3 and 4 shows HindIII digested clones $1,2,3$ and 4 respectively. Molecular size markers (GIBCO/BRL) are shown to the right.

\section{CAGGGATCCGCGGCTATGCTTCCTGTGAG and 3'AvrF-} ACGTCCTAGGTTAACAGACGCCACCACG. The $1.1 \mathrm{~kb}$ PCR product obtained was purified, digested with BamHI and $A v r I I$ and cloned into BglII and $A v r I I$ digested plasmid pEF-HLT-FG. The identity of the final construct pTcF (9.2 kb) (Figure 1B) was done by sequencing and restriction analysis.

The construct $\mathrm{pTcF}$ was linearized by digestion with respective restriction enzymes and purified by electrophoresis on low melting agarose before transfection. Mainly two steps were involved in the creation of tetracycline regulatable single knockouts of ORFF. As a first step, the tet repressor (TetR) was introduced into the tubulin locus (selectable marker was puromycin) (Figure 1A). 


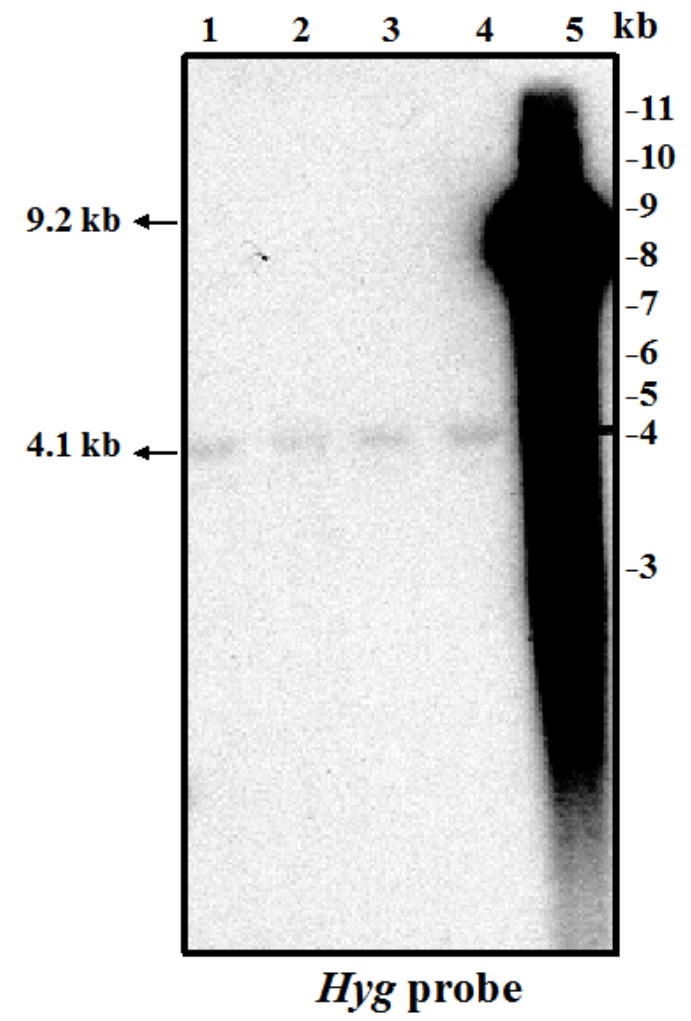

Fig. 4: Genomic Southern blot analysis of AG83/TetR/ $\Delta \mathrm{F}, \mathrm{rPF} \quad$ clones using hygromycin probe (1048-bp). Genomic DNA was isolated from AG83/TetR/ $\Delta \mathrm{F}, \mathrm{rPF}$ clones and was digested with ClaI and NotI. The DNA was size fractionated on $1 \%$ agarose gel and blotted to nylon membrane. The filters were hybridized with ${ }^{32} \mathrm{P}$-labeled $H Y G$ probe. Filters were washed and exposed overnight to X-ray film. Lanes 1, 2, 3 and 4 shows ClaI and NotI digested clones 1, 2, 3 and 4 respectively. Lane 5 shows the linearized pTcF plasmid (positive control). Molecular size markers (GIBCO/BRL) are shown to the right.

The second step was to replace the first allele of ORFF with the tetracycline inducible allele of ORFF (selectable marker used was hygromycin) (Figure 1B). Figure 2 shows the schematics of the ORFF genomic region before and after the targeted single allele deletion of ORFF gene. pFHLTG construct was used to check for the functionality of Tetracycline repressor (TetR) introduced into tubulin locus of $L$. donovani strain AG83 using transient luciferase assay ${ }^{[1]}$.
DNA transfection: Methods of electroporation and plating of L. donovani have been described previously ${ }^{[25]}$. Briefly, $4 \times 10^{7}$ promastigotes of $L$. donovani in $0.4 \mathrm{ml}$ electroporation buffer $(21 \mathrm{mM}$ HEPES, $137 \mathrm{mM} \mathrm{NaCl}, 5 \mathrm{mM} \mathrm{KCl}, 0.7 \mathrm{mM} \mathrm{NaH}{ }_{2} \mathrm{PO}_{4}$, $6 \mathrm{mM}$ glucose, $\mathrm{pH}$ 7.4) were transfected with $5 \mu \mathrm{g}$ of DNA by electroporation at $480 \mathrm{~V}, 500 \mu \mathrm{F}, 13 \Omega$ (BTX Electro Cell Manipulator 600). Cells were cultured for $\sim 24 \mathrm{~h}$ in M199 media containing 10\% FCS before spreading on plates containing $0.7 \%$ Seaplaque GTG agarose (FMC Bioproducts) in M199 media (with 10\% FCS) with puromycin $(45 \mu \mathrm{M})$ or hygromycin $(25$ $\mu \mathrm{g} / \mathrm{ml})$ to select for single colonies. Wild type AG83 was maintained in M199 (10\% FCS), ORFF single knockout (AG83/tetR/ $\Delta \mathrm{F}, \mathrm{rPF}$ ) was maintained in M199 (10\% FCS) with hygromycin $(25 \mu \mathrm{g} / \mathrm{ml})$ and tetracycline $(10 \mu \mathrm{g} / \mathrm{ml})$.

Luciferase assay: Approximately $10^{5}$ or $10^{6}$ cells were lysed and assayed for luciferase activity using a Monolight luminometer (Analytic Luminescence Laboratory) following manufacturer's instructions. The luciferase activities were measured for $10 \mathrm{~s}$. Experiments were repeated thrice and the data presented are representative.

Nucleic acid isolation and hybridization analysis: Genomic DNA was extracted from Leishmania using earlier reported method. ${ }^{[26]}$. Late log-phase promastigotes were collected by centrifugation, washed in $1 \mathrm{x}$ phosphate buffered saline (PBS), resuspended in EA solution (0.01 M Tris, $\mathrm{pH} 8.0,0.25 \mathrm{M} \mathrm{NaCl}$, $0.005 \% \mathrm{NP}-40$ ) and incubated for $5 \mathrm{~min}$ at room temperature. Lysates were spun at $13,000 \mathrm{x} \mathrm{g}$ for 15 min and the pellets resuspended in EB solution $(0.01 \mathrm{M}$ Tris, $\mathrm{pH}$ 8.0, 0.01M EDTA, 0.5\% SDS, $50 \mu \mathrm{g} / \mathrm{ml}$ proteinase $\mathrm{K}$ ) and incubated on ice for $5 \mathrm{~min}$. The DNA was extracted sequentially with equal volumes of phenol:chloroform:isoamylalcohol (25:24:1) and chloroform:isoamyl alcohol $(24: 1)$, then precipitated at $-20^{\circ} \mathrm{C}$ with $0.3 \mathrm{M}$ sodium acetate and 1 volume of isopropanol. The DNA pellet was collected by centrifugation and resuspended in TE $(10 \mathrm{mM}$ Tris, $\mathrm{pH}$ 8.0, $1 \mathrm{mM}$ EDTA). $10 \mu \mathrm{g}$ of DNA was digested with appropriate restriction enzymes and separated by $1 \%$ agarose gel electrophoresis, stained with ethidium bromide, and transferred to nylon membranes as described previously ${ }^{[15]}$.

Total RNA was isolated from Leishmania using Trireagent (Sigma) according to manufacturer's 
$\mathbf{A}$

B
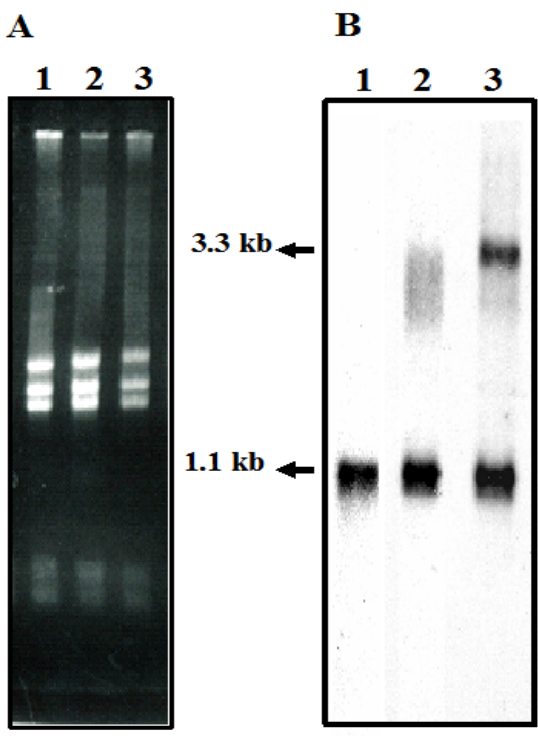

ORFF probe

Fig. 5: Northern blot analysis of $A G 83 / \mathrm{TetR} / \Delta \mathrm{F}, \mathrm{rPF}$ (SKO) using ORFF specific probe in the absence and presence of tetracycline. Total RNA was isolated from wild type, AG83/TetR/ $\Delta \mathrm{F}, \quad \mathrm{rPF}$ cells grown in the presence and absence of tetracycline. $10 \mu \mathrm{g}$ of total RNA was run on a $1 \%$ formaldehyde gel and transferred to nylon membrane. The filters were hybridized with ${ }^{32} \mathrm{P}$-labeled $O R F F$ probe. A: Ethidium bromide stained formaldehyde gel. B: Northern blot probed with $O R F F$ probe. Lane 1: total RNA from AG83 (wild type cells). Lane 2: RNA isolated from SKO cells grown in the absence of tetracycline. Lane 3: total RNA isolated from single knockout cells grown in the presence of tetracycline. All the 3 lanes show the presence of the wild type copy of ORFF $(1.1 \mathrm{~kb})$ in the Northern blot. The SKO cells in the presence of tetracycline showed the presence of $3.3 \mathrm{~kb}$ inducible copy of ORFF besides $1.1 \mathrm{~kb}$ wild type allele.

instructions. Approximately $10 \mu \mathrm{g}$ of total RNA was separated on MOPS/formaldehyde agarose gel and transferred to nylon membrane and cross-linked to the filters with a Stratagene UV-cross linker (Stratagene) according to manufacturer's instruction.

Blots were hybridized with radiolabeled probes prepared by Prime-a-Gene random prime synthesis with Klenow DNA polymerase and $\alpha\left[{ }^{32} \mathrm{P}\right] \mathrm{dCTP}$. The EK1 probe ${ }^{[27,18]}$ corresponding to ORFF of LD1 locus was used for hybridization. Hybridization was carried out for $16-18 \mathrm{~h}$ at $42^{\circ} \mathrm{C}$. The TetR probe $(647 \mathrm{bp})$ was obtained by digesting pTupactet with BamH1 and SpeI. Hygromycin probe (1048 bp) was obtained from $\mathrm{pTcF}$ by digesting the plasmid with SpeI and BamHI. The probe specific for ORFF gene was obtained by the restriction digestion of $\mathrm{pTcF}$ using enzymes $\mathrm{KpnI}$ and AvrII.

Growth studies: Growth profile of AG83, single ORFF targeted mutants of AG83 in the presence and absence of tetracycline was studied by inoculating stationary phase cells in M199 (10\% FCS) at a density of $\sim 2 \times 10^{6}$ cells $/ \mathrm{ml}$. Cells were counted at $24 \mathrm{~h}$ intervals using hemocytometer.

In vitro macrophage infection: J774.A1 murine macrophage cells were plated in $35 \mathrm{~mm}$ tissue culture dishes at a density of $1 \times 10^{5}$ per dish. In vitro infection of J774A.1 macrophages was done with $1 \times 10^{6}$ labeled promastigotes of AG83 and ORFF single allele deletion mutants in the presence and absence of tetracycline. Parasites were labeled by resuspending them at a density of $1 \times 10^{7}$ cells $/ 2 \mathrm{ml}$ M199 medium having $8 \mu \mathrm{Ci} / \mathrm{ml}\left[{ }^{3} \mathrm{H}\right]$-Thymidine (specific activity $=$ $89.4 \mathrm{Ci} / \mathrm{mmol}$, New England Nuclear) and incubated for $3 \mathrm{~h}$ at $22^{\circ} \mathrm{C}$. After the incubation, monolayers were washed twice with $1 \mathrm{ml}$ PBS to remove unbound parasites and placed on ice to loosen adherent cells from the plates. Samples were processed for scintillation counting by adding $500 \mu \mathrm{l}$ of $0.5 \%$ Triton $\mathrm{X}-100$ per dish and transferring the solution onto GF/C filters. Filters were then dried in the oven, soaked in scintillation fluid $(5 \mathrm{ml})$ and counting was done using a Packard Tri-Carb liquid scintillation counter.

\section{RESULTS}

Introduction of tetracycline repressor (TetR) into tubulin locus of $\boldsymbol{L}$. donovani: $L$. donovani (AG83) cells which have a single copy of ORFF

Table 1: Transient luciferase assay to estimate the regulation of TetR in the tubulin locus using tetracyline as the inducer

\begin{tabular}{llll}
\hline \multicolumn{1}{c}{ Population } & \multicolumn{2}{l}{ RLU per $10^{6}$ cells } & $\begin{array}{c}\text {-fold } \\
\text { increase }\end{array}$ \\
\cline { 2 - 3 } & -tet & ttet & \\
\hline & & & \\
Clone 1 & 408 & 63985 & 157 \\
Clone 2 & 2472 & 85913 & 35 \\
Clone 3 & 2980 & 65946 & 22 \\
Clone 4 & 4369 & 59812 & 14 \\
413 cell line(C) & 55277 & 1154569 & 21 \\
& & & \\
\hline
\end{tabular}

$4 \times 10^{7}$ cells ( 4 clones of $L$. donovani strain AG83 positive for TetR integration into tubulin locus and $L$ donovani cell line 4.1.3) were electroporated using $5 \mu \mathrm{g}$ of pFHLTG (which has a Luciferase gene driven by a regulatable promoter). After electroporation, half of the cells were grown in the 
presence of tetracycline and the other half in the absence of tetracycline. Luciferase assay was done on the next day. As a control, $L$ donovani cell line 413 , expressing tet $R$ from $\alpha$-tubulin locus of LSB51.1 was also used. The Luciferase activities are given as average relative light units (RLU) from duplicate samples.

gene at the LD1 locus on chromosome 35 were used for these studies. Plasmid pTupactet, linearized using $K p n \mathrm{I}$ and NotI. was electroporated into $4 \times 10^{7} \mathrm{~L}$. donovani (AG83) cells. As a control, uncut plasmid (pTupactet) was also electroporated into $L$. donovani cells. Cells growing in the presence of puromycin were cloned on M199/10\% FCS/puromycin/semisolid agar plates. 30 colonies were obtained using one microgram of linearized pTupactet. 22 colonies from this group were picked up and grown in $1 \mathrm{ml}$ of $\mathrm{M} 199 / 10 \%$ FCS/puromycin (Group 1). Approximately 200 colonies were obtained using nonlinearized pTupactet. 8 colonies from this group were picked up and grown in $1 \mathrm{ml}$ of M199/10\% FCS /puromycin (Group 2). Slowly the culture was scaled up to $10 \mathrm{ml}$. Four clones from Group 1 and two clones from Group 2 were chosen for Southern analysis. Genomic DNA from the clonal transfectants was digested with HindIII and transferred to nylon membrane according to the protocol given in the materials and methods section. The blot was hybridized with the TetR probe (647-bp). Clones 1, 2, 3 and 4 (obtained by transfecting with linearized pTupactet) showed a 4.6-kb band upon hybridization with TetR probe (Figure 3, Lanes 1, 2, 3 and 4). This is consistent with the integration of TetR into the tubulin locus. The genomic DNA from clones transfected with circular plasmid showed a 7.6-kb band indicating their existence as an episome within the Leishmanial cells (data not shown). Genomic DNA from non-transfected AG83 cells did not hybridize with the TetR probe (data not shown). Thus it was confirmed that $T e t R$ is integrated into the tubulin locus.

Regulation of TetR in the tubulin locus using tetracyline as the inducer: In order to check whether $T e t R$ is functional in the genome of cell lines where it was integrated, transient luciferase assay was done. As a control, $L$ donovani cell line 4.1.3, expressing Tet $R$ from $\alpha$-tubulin locus of LSB-51.1 ${ }^{[1]}$ was also used. $4 \times$ $10^{7}$ cells ( 4 clones of $L$. donovani strain AG83 positive for Tet $R$ integration into tubulin locus and $L$ donovani cell line 4.1.3) were electroporated with pFHLTG construct (which has a Luciferase gene driven by a regulatable rRNA promoter) ${ }^{[6]}$. After electroporation, half of the cells were grown in the presence of tetracyclin and the other half in the absence of tetracycline. Luciferase assay was done on the next day. The results of the assay are given in Table 1.

All the clones showed more than 10-fold regulation of luciferase activity in the presence of tetracycline (compared to the cell population where no tetracycline was added). Thus TetR was functional and could be regulated by tetracycline added externally to the media. The transformed AG83 clone (clone 2) expressing TetR in the tubulin locus was named as AG83/tetR and was used for the further studies. Henceforth, AG83/tetR cells are depicted as wild type in all the experiments.

Replacement of one allele of ORFF with the tetracycline regulatable allele of ORFF to create AG83/tetR/ $\Delta F$, PPF (SKO, Single knockout): First wild type allele of ORFF was knocked out using the construct pTcF. AG83/tetR cells were transfected with linearized pTcF (digested with BstXI and HindIII). The transfectants were plated on M199/10\%/hygromycin/tetracycline plates. Four clones were obtained. Total genomic DNA from all the clones was digested with ClaI and NotI and analysed by Southern blot. As a positive control, linearized pTcF $(9.2 \mathrm{~kb})$ was also used. Figure 4 shows the corresponding Southern blot after

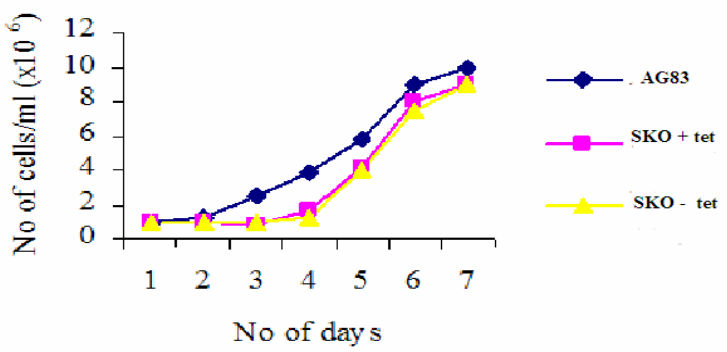

Fig. 6: Growth rate analysis of wild type and single knockout clone of Leishmania. Growth profile of wild type, single knockout in the presence of tetracycline (SKO + tet) and single knockout clone in the absence of tetracycline (SKO-tet) was done by inoculating stationary phase cells in M199(10\% FCS) at a density of $2 \times 10^{6}$ cells $/ \mathrm{ml}$. Cells were counted at $24 \mathrm{~h}$ intervals using a hemocytometer. 


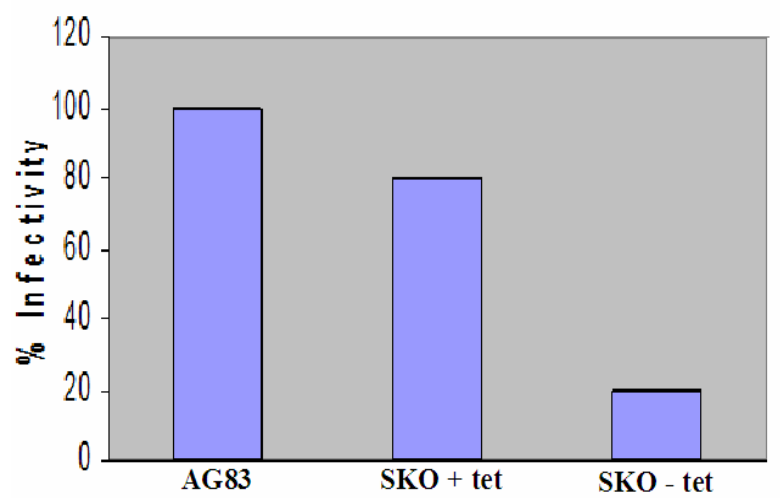

Fig. 7: In vitro infection of J774A.1 macrophages was done with 1 x $10^{6}$ labeled ( ${ }^{3} \mathrm{H}$-thymidine) promastigotes of $\mathrm{AG} 83$ and $\mathrm{AG} 83 / \mathrm{TetR} / \Delta \mathrm{F}, \mathrm{rPF}$ (SKO) in the presence and absence of tetracycline. Parasites $\left(10^{7}\right.$ cells/ $2 \mathrm{ml}$ M199/10\% FCS) were labeled with $8 \mu \mathrm{Ci} / \mathrm{ml}$ $\left[{ }^{3} \mathrm{H}\right]$-Thymidine and incubated for $3 \mathrm{~h}$ at $22^{\circ} \mathrm{C}$. After the incubation, monolayers were washed twice with $1 \mathrm{ml}$ PBS and layered with Triton X $100(1 \%)$ to lyse the macrophages. Samples were processed for scintillation counting by transferring the solution onto GF/C filters. Filters were then dried in the oven, soaked in scintillation fluid $(5 \mathrm{ml})$ and counted. The results are given as percentage infectivity. The results shown here represent the mean of two experiments.

hybridization with hygromycin probe. Lanes 1, 2, 3 and 4 represent clones $1,2,3$ and 4 respectively. Lane 5 shows the linearized $\mathrm{pTcF}$ control. All the clones showed the expected band size of $4.1 \mathrm{~kb}$ on hybridization with hygromycin probe (Figure 4). One of the clones (clone 2, Lane 2) was named as AG83/tetR/ $\triangle F$, rPF (SKO) and used for further studies.

ORFF expression in AG83/tetR/ $\Delta F, r P F$ (SKO) in the presence and absence of tetracycline: In order to check the expression of ORFF in AG83/tetR/ $\triangle F, r P F$ in the presence and absence of tetracycline, AG83/tetR/ $\triangle \mathrm{F}, \mathrm{rPF}$ mutant was grown in the presence and in the absence of tetracycline. Total RNA was isolated from log phase culture of the clone. Northern analysis was done using $O R F F$ specific DNA probe. The ORFF probe was obtained by the restriction digestion of pTcF using enzymes KpnI and $A v r I I$. Figure $5 \mathrm{~A}$ shows the ethidium bromide stained RNA gel. Lane 1 represents wild type (AG83), lane 2 shows SKO clone grown in the absence of tetracycline and lane 3 shows the same SKO clone grown in the presence of tetracycline. RNA isolated from wild type cells (Lane 1) and SKO grown in the absence (Lane 2) and presence of tetracycline (Lane 3) showed the expression of the wild type allele $(1.1 \mathrm{~kb})$ (Figure $5 \mathrm{~B})$. Apart from the wild type allele, the single knockout clone in the presence of tetracycline also showed the expression of the inducible allele of ORFF $(3.3 \mathrm{~kb})$, which was absent in the wild type and also SKO clone in the absence of tetracycline (Figure 5B).

Growth rate analysis: The doubling time of wild type AG83 was $36 \mathrm{~h}$ and that of single knockout was $40 \mathrm{~h}$ in the presence of tetracycline. The single knockout mutants did not show any difference in the doubling time in the presence and absence of tetracycline (Figure 6).

In vitro infectivity assay: In vitro infection of J774A. 1 macrophages was done with $1 \times 10^{6}$ labeled promastigotes of $\mathrm{AG} 83$, and $\mathrm{AG} 83 /$ tetR/ $\triangle \mathrm{F}, \mathrm{rPF}$ in the presence and absence of tetracycline. Infectivity into macrophages reduced by almost $80 \%$ with single knockout clone in the absence of tetracycline compared to the wild type AG83 and $A G 83 /$ tetR/ $\Delta F, r P F$ in the presence of tetracycline.

\section{DISCUSSION}

The targeted gene deletion of ORFF gene from the LD1 locus of Leishmania donovani was accomplished by adapting the tetracycline responsive inducible promoter system in the ORFF genomic region. As the first step, the tetracycline repressor (TetR) was introduced into the tubulin locus of Leishmania donovani (AG83) to create AG83/tetR. The next step involved the cloning of ORFF gene under the control of tetracycline regulatable rRNA promoter in the reverse orientation compared to the transcription of the selectable marker gene $(H Y G)$. This construct was used to replace the first wild type allele of ORFF gene at the LD1 locus.

Southern analysis of the single targeted mutants using ORFF specific probe showed that all the clones possessed the inducible copy of ORFF along with the wild type copy. We were successful in generating tetracycline regulatable single knock out mutants of ORFF in Leishmania donovani strain AG83.

The functional study in the context of parasite's growth carried out in the single knockouts in the presence of 
tetracycline showed that the growth rate of the single targeted knockouts was not different from the wild type and the single targeted knockouts grown in the absence of tetracycline

The in vitro infectivity studies have shown that the infectivity, more precisely the attachment of the recombinant parasites to the macrophages was considerably reduced $(80 \%$ in the absence of tetracycline) when compared to the wild type.

This study, even though not conclusive, shows the possibility of generating functional tetracycline regulatable single knock out mutants of Leishmania donovani. The present study also demonstrates that ORFF gene might be an important gene for the survival of the parasite and it may be involved in various functions like attachment of the macrophages to Leishmania.

\section{ACKNOWLEDGEMENTS}

This work is supported by the Department of Science and Technology, Government of India, New Delhi, India to RMB. Manju Jain is supported by a grant from the Council of Scientific and Industrial Research, New Delhi, India. BS was supported by a grant from the University Grants Commission, New Delhi, India. BS was also supported by a training grant (International Training and Research in Emerging Infectious Diseases, ITREID) from Fogarty International Centre, USA.

\section{REFERENCES}

1. Yan, S., P.J. Myler and K. Stuart, 2001. Tetracycline regulated gene expression in Leishmania donovani. Mol. Biochem. Parasitol., 112: 61-69.

2. Dingermann, T., U. Frank-Stoll, H. Werner, A. Wissmann, W. Hillen, M. Jacquet and R. Marschalek, 1992. RNA polymerase III catalysed transcription can be regulated in Saccharomyces cerevisiae by the bacterial tetracycline repressoroperator system. EMBO J., 11: 1487-1492.

3. Gatz, C. and P.H. Quail, 1988. Tn10-encoded tet repressor can regulate an operator-containing plant promoter. Proc. Natl. Acad. Sci. USA, 85: 13941397.

4. Wirtz, E. and C. Clayton, 1995. Inducible gene expression in trypanosomes mediated by a prokaryotic repressor. Science, 268: 1179-1183.

5. Clayton, C.E., 1999. Genetic Manipulation of Kinetoplastida. Parasitol. Today, 15: 373-378.
6. Yan, S., S. Martinez-Calvillo, A. Schnaufer, S. Sunkin, P.J. Myler and K. Stuart, 2002. A lowbackground inducible promoter system in Leishmania donovani. Mol. Biochem. Parasitol., 119: 217-223.

7. Gay, L.S., M.E. Wilson and J.E. Donelson, 1996. The promoter for the ribosomal RNA genes of Leishmania chagasi. Mol. Biochem. Parasitol., 77: 193-200.

8. Tyler-Cross, R.E., S.L. Short, L.M. Floeter-Winter and G.A. Buck, 1995. Transient expression mediated by the Trypanosoma cruzi rRNA promoter. Mol. Biochem. Parasitol., 72: 23-31.

9. Uliana, S.R.B., W. Fischer, V.A. Stempliuk and L.M Floeter-Winter, 1996. Structural and functional characterization of the Leishmania amazonensis ribosomal RNA promoter. Mol. Biochem. Parasitol., 76: 245-255.

10. Yan, S., M.J. Lodes, M. Fox, P.J. Myler and K. Stuart, 1999. Characterization of the Leishmania donovani ribosomal RNA promoter. Mol. Biochem. Parasitol., 103: 197-210.

11. Zomerdijk, J.C.B.M., R. Kieft, P.G. Shiels and P. Borst, 1991. Alpha-amanitin-resistant transcription units in trypanosomes: A comparison of promoter sequences for a VSG gene expression site and for the ribosomal RNA genes. Nucl. Acids Res., 19: 5153-5158.

12. Yarranton, G.T., 1992. Inducible vectors for expression in mammalian cells. Curr. Opin. Biotechnol., 3: 506-511.

13. Ghosh, A., R. Madhubala, P.J. Myler and K.D. Stuart, 1999. Leishmania donovani characterization and expression of ORFF, a gene amplified from the LDI locus. Exp. Parasitol. 93: 225-230.

14. Lemley, C., S. Yan, V.S. Dole, R. Madhubala, M.L. Cunningham, S.M. Beverley, P.J. Myler and K.D. Stuart, 1999. The Leishmania donovani LD1 locus gene ORFG encodes a biopterin transporter (BT1). Mol. Biochem. Parasitol., 104: 93-105.

15. Lodes, M.J., G. Merlin, T. Devos, A. Ghosh, R. Madhubala, P.J. Myler and K. Stuart, 1995. Increased expression of LD1 genes transcribed by RNA polymerase I in Leishmania donovani as a result of duplication into the rRNA gene locus. Mol. Cell. Biol., 15: 6845-6853.

16. Myler, P.J., M.J. Lodes, G. Merlin, T. de Vos and K.D. Stuart, 1994a. An amplified DNA element in Leishmania encodes potential integral membrane and nucleotide-binding proteins. Mol. Biochem. Parasitol., 66: 11-20. 
17. Raj, V.S., A. Ghosh, V.S. Dole, R. Madhubala, P.J. Myler and K.D. Stuart, 1999. Serodiagnosis of leishmaniasis with recombinant ORFF antigen. Am. J. Trop. Med. Hyg., 61: 482-487.

18. Tripp, C.A., W.A. Wisdom, P.J. Myler and K. Stuart, 1992. A multicopy, extrachromosomal DNA in Leishmania infantum contains two inverted repeats of the $27.5 \mathrm{~kb}$ LD1 sequence and encodes numerous transcripts. Mol. Biochem. Parasitol. 55: 39-50.

19. Dole, V.S., V.S. Raj, R. Madhubala, P.J. Myler and K.D. Stuart, 2001. Immunization with recombinant LD1 antigens protects against experimental leishmaniasis. Vaccine, 19: 423-430.

20. Sukumaran, B., P. Tewary, S. Saxena and R. Madhubala, 2003. Vaccination with DNA encoding ORFF antigen confers protective immunity in mice infected with Leishmania donovani. Vaccine, 21: 1292-1299.

21. Tewary, P., J. Pandyan, B. Sukumaran and R. Madhubala, 2004a. Vaccination with Leishmania soluble antigen and immunostimulatory oligodeoxynucleotides inducs specific immunity and protection against Leishmania donovani infection. FEMS Immunol. Med. Microbiol., 42: 241-248.
22. Tewary, P., B. Sukumaran, S. Saxena and R. Madhubala, 2004b. Immunostimulatory Oligodeoxynucleotides are potent enhancers of specific immunity in mice immunized with recombinant ORFF leishmanial antigen. Vaccine, 22: 3053-3060.

23. Tewary, P., M. Jain, M. Sahani, S. Saxena and R. Madhubala, 2005. A heterologous Prime Boost Vaccine regime ORFF DNA and recombinant ORFF Protein Confers Protective Immunity against Experimental Visceral Leishmaniasis in Murine Mouse Model. J. Infect. Dis., 191: 21302137.

24. Gueiros-Filh, F.J. and S.M. Beverley, 1997. Transkingdom transposition of the Drosophila element mariner within the protozoan Leishmania. Science, 276: 1716-1719.

25. Kapler, G.M., C.M. Coburn and S.M. Beverley, 1990. Stable transfection of the human parasite Leishmania major delineates a 30- kilobase region sufficient for extra chromosomal replication and expression. Mol. Cell. Biol., 10: 1084-1094.

26. Bellafato, V. and G.A.M. Cross, 1989. Expression of a bacterial gene in a trypanosomatid protozoan. Science, 244: 1167-1169.

27. Myler, P.J., G.M. Venkataraman, M.J. Lodes and K.D. Stuart, 1994b. A frequently amplified region in Leishmania contains a gene that is conserved in prokaryotes and eukaryotes. Gene, 148: 187-193 